\title{
INDICADORES DE EVALUACIÓN DEL DESEMPEÑO AMBIENTAL EN UNA ORGANIZACIÓN CUBANAฯ
}

\author{
INDICATORS OF EVALUATION OF ENVIRONMENTAL PERFORMANCE IN \\ A CUBAN ORGANIZATION
}

\author{
Roani Ladislá Miranda Cuéllar ${ }^{1, \star}$, Yipsian de la Caridad Betancourt García², \\ Lourdes Santos Planas ${ }^{3}$
}

\section{RESUMO}

La supervivencia de la humanidad es uno de los problemas ambientales más grande que enfrenta el planeta Tierra hoy en día, por lo que la necesidad de preservar y contribuir al cuidado del medio ambiente adquiere un significado especial. Para detener la explotación acelerada e irracional de los recursos naturales, se elaboran estrategias y se realizan diversas tareas complejas. Con la construcción y mantenimiento de indicadores, estas acciones pueden ser monitoreadas y controladas. El objetivo de este trabajo es contribuir a la mejora de la evaluación del desempeño ambiental en una organización cubana. Se diseñaron los Pasos metodológicos para el diagnóstico del comportamiento ambiental de la organización y se aplicaron diferentes herramientas y técnicas que permitieron analizar el estado actual de la evaluación del desempeño ambiental en la organización. Se detectaron deficiencias en la política ambiental, objetivos, metas e indicadores. Se reformuló la política ambiental, se enriquecieron los criterios de evaluación de desempeño ambiental, se crearon nuevos objetivos, metas e indicadores que se armonizaron entre sí y se calcularon de acuerdo con la información disponible. Los resultados de la política ambiental se cuantificaron de manera resumida y se determinó el nivel de desempeño ambiental que permitió a la organización adoptar nuevas decisiones.

Palabras clave: Gestión ambiental, indicadores de evaluación del desempeño ambiental, información ambiental, toma de decisiones, método Delphi.

\footnotetext{
\&Fue presentada una versión resumida en el X Simposio Iberoamericano sobre Medio Ambiente durante la XIX Convención Científica de Ingeniería y Arquitectura, celebrada en La Habana, Cuba del 2 ( al 30 noviembre del 2018

${ }^{1}$ Departamento Informática Empresarial, Facultad de Ingeniería Industrial, Universidad Tecnológica de La Habana “José Antonio Echeverria”, La Habana, Cuba. orcid.org/0000-0002-0781-6155 ${ }^{2}$ Ministerio de la industria Alimentaria. Cuba. orcid.org/0000-0003-3793-1637

${ }^{3}$ Departamento Informática Empresarial, Facultad de Ingeniería Industrial, Universidad Tecnológica de La Habana "José Antonio Echeverria", La Habana, Cuba. orcid.org/0000-0003-1278-1548
} 


\section{ABSTRACT}

The survival of humanity is one of the environmental problems, the biggest one facing planet Earth today, which is why the need to preserve and contribute to the care of the environment takes on special significance. To stop the accelerated and irrational exploitation of natural resources, strategies are drowning up and various complex tasks were carried out. With the construction and maintenance of indicators, these actions can be monitoring and controlling. The objective of this work is to contribute its environmental performance evaluation in a Cuban organization. Methodological Steps to diagnosis the environmental behavior of the organization was designing and different tools and techniques were applying to analyze the current state of environmental performance evaluation in the organization. A was made of the according to the methodological steps and the environmental indicators that are implemented were identified, as well as the environmental performance criteria considered by the interested parties. Insufficiencies in environmental policy, objectives, goals, and indicators were detecting. The environmental policy was reformulating; new objectives new goals and indicators harmonized with each other we are creating and calculated according to the available information. The results of the environmental policy were quantified in a summarized manner and were determined the level of environmental performance which enabled the organization to adopt new decisions.

Keywords: Environmental management, environmental performance evaluation indicators, environmental information, decision-making, Delphi method.

\section{INTRODUCCIÓN}

La sociedad actual sobreexplota los recursos naturales, provoca la extinción de especies, deteriora los suelos, desaparece bosques, fragmenta hábitats, transforma el estado de los recursos hídricos en no renovables, entre otras muchas acciones que agravado por los perceptibles efectos del cambio climático provoca que el medio ambiente se deteriore continuamente. Ha contaminado el aire, el suelo y el agua, en esencia se ha hipotecado el futuro (Miranda Cuéllar \& Fonden Calzadilla, 2019).

No obstante, ha sido reconocida la necesidad de preservar el medio ambiente: Conferencia de Naciones Unidas sobre Medio Ambiente y Desarrollo (ONU, 1992; ONU, 2002; ONU, 2012; ONU, 2015); así como múltiples convenios. Por esto, el compromiso de los Estados trasciende a todas las organizaciones, independientemente de su tamaño y su objeto social, siendo su expresión más evidente la gestión ambiental, que unida a la información relacionada con el medio ambiente, constituyen tema de primer orden en las agendas internacionales.

Ese compromiso se evidencia en los informes que muestran las acciones que las organizaciones y los gobiernos llevan a cabo a favor del medio ambiente, utilizando múltiples iniciativas voluntarias de organismos internacionales, entre las que se encuentran: Organización de Cooperación y Desarrollo Económicos (OCDE), La Iniciativa Global para hacer Informes de Sostenibilidad (GRI por sus siglas en ingles), los Indicadores de Desarrollo del Milenio, hoy Objetivos de Desarrollo Sostenible (ODS) y la Iniciativa Latinoamericana y Caribeña para el Desarrollo Sostenible (ILAC), de la cual Cuba es miembro. Estas iniciativas constituyen vehículos de información ambiental que se sustentan en indicadores y tienen puntos en común con diferentes niveles de integración. A continuación, se abordan algunos 
ejemplos de su quehacer.

En consonancia con estas alternativas se realizó la evaluación del desempeño ambiental en Perú, preparada por la Comisión Económica para América Latina y el Caribe (CEPAL) y la OCDE, con el objetivo de ayudar al país a evaluar los avances logrados en la consecución de sus objetivos ambientales, así como enriquecer y asegurar la constancia del diálogo de políticas, promover una mayor rendición de cuentas y una concientización más profunda entre los actores que contribuyen al desarrollo económico y social (CEPAL/OCDE, 2017).

Los informes o memorias de sostenibilidad bajo la plataforma GRI están enfoque a reflejar cómo una organización se orienta hacia el desarrollo sostenible puesto que en las guías para la elaboración de memorias de sostenibilidad proponen que estén conformadas con información sobre la incidencia de las organizaciones en el medio ambiente, la sociedad y la economía (GRI, 2013).

En tal sentido, Dechkova y Miranda (2014) responden a la pregunta si se debe emitir un informe de sostenibilidad o no, empleando la lógica difusa compensatoria y estructuran las actividades de toma de decisiones a través de tres aspectos: la preparación, la conveniencia para emitir los informes de sostenibilidad y su utilidad (Dechkova \& Miranda, 2014). Por su parte Rodríguez y Ríos (2016) en su investigación catalogan a las organizaciones colombianas de acuerdo a su desempeño bajo la metodología GRI, G4 (Rodríguez Guerra \& Ríos-Osorio, 2016).

La Agenda 2030 para el Desarrollo Sostenible con sus 17 Objetivos (ODS), 169 metas y sus 304 indicadores, ayuda a evaluar el punto de partida de los países de la región, a analizar y formular los medios para alcanzar esta nueva visión del desarrollo sostenible. Esta constituye una herramienta de planificación y seguimiento para los países tanto a nivel nacional como local, hacia un desarrollo sostenido, inclusivo y en armonía con el medio ambiente, a través de políticas públicas e instrumentos de presupuesto, monitoreo y evaluación (ONU, 2018).

Para dar cumplimiento a la Agenda 2030 se realiza la adaptación de las metas a los contextos nacionales, la selección de indicadores de seguimiento y la elaboración de fichas técnicas, lo que contribuye a establecer bases sólidas para el monitoreo posterior. El resultado del proceso de implementación culmina con el Informe Voluntario Nacional como los de Argentina, España y Cuba. (Argentina, 2018; España, 2018; Cuba, 2019).

La ILAC estableció un conjunto medular de estadísticas e indicadores ambiental es que permiten monitorear el progreso hacia las metas ambientales, sin embargo, la publicación realizada en el 2004: Iniciativa Latinoamericana y Caribeña para el Desarrollo Sostenible: Indicadores de seguimiento (ONU, 2004), plantea que en la región de América Latina y el Caribe el tema ambiental ocupa un lugar importante en las agendas políticas, donde la construcción de información ambiental es básica y esencial en la planeación y el proceso de toma de decisiones, pero la carencia de estadísticas ambientales pertinentes, relevantes y sistemáticas ha retrasado la construcción de indicadores de seguimiento de esta Iniciativa.

De igual forma, el PNUMA, en el Resumen para los responsables de la formulación de políticas en las conclusiones del quinto informe Perspectivas del Medio Ambiente Mundial (GEO 5), afirma que la falta de datos cronológicos confiables y sistemáticos sobre el estado del medio ambiente obstaculiza sobremanera la posibilidad de aumentar la eficacia de las políticas y programas (PNUMA, 2012).

En el contexto actual, la ILAC ha establecido sus indicadores para el Desarrollo Sostenible y lleva a cabo acciones ante los desafíos en la disponibilidad y uso de información a la hora de 
dar seguimiento al estado del ambiente (GAETANI, 2018).

De igual manera, las Pymes están conscientes de ello y de la necesidad de incorporarse al mercado internacional, que tiene como requisito obligatorio demostrar un sólido desempeño ambiental (Corella, 2013; Wong et al., 2012), en correspondencia con las expectativas de las partes interesadas y la transparencia de sus informaciones (GRI, 2013; ISO-26000, 2010; Dechkova \& Miranda, 2014). Para ello se hacen corresponder sus evaluaciones con las iniciativas y en consonancia con normas internacionales como la norma 14001 de gestión ambiental, que considera indicadores.

La representación medible de la condición o el estado de las operaciones, la gestión, o las condiciones (ONN, 2015) son los indicadores en los que se sustenta la información como recurso intangible. Un indicador ambiental es una medida cuantitativa y/o cualitativa que expresa el cumplimiento de requisitos legales, objetivos ambientales y/o compromisos internacionales (Miranda Cuéllar et al., 2016), en un momento y en un espacio determinado, adquiriendo así un gran valor como herramienta en la evaluación de procesos y la toma de decisiones en torno a los problemas ambientales (Junta de Andalucía, 2014).

Para llevar a cabo la Evaluación del Desempeño Ambiental (EDA) puede ser utilizada la norma 14031, ya que es una herramienta de la gestión ambiental que potencia la retroalimentación de la gestión y el seguimiento de los resultados ambientales alcanzados por las organizaciones que la aplican. Esta norma es un proceso y una herramienta de gestión interna diseñada para proporcionar información fiable y verificable continuamente a la dirección, que posibilita determinar si el desempeño ambiental está cumpliendo con los criterios establecidos. Para ello se seleccionan indicadores, se realiza la recopilación y el análisis de datos, la evaluación de la información comparada con los criterios de desempeño ambiental, los informes y comunicaciones, las revisiones periódicas, y las mejoras de este proceso (ONN, 2005). Esta norma no cuantifica de manera resumida los resultados de la política ambiental de una organización y no establece niveles de desempeño ambiental, por lo que cada organización implementa esta norma y la adapta a su contexto. Entonces, en este trabajo se llevan a cabo un conjunto de pasos que facilitan su cuantificación.

Para evitar evaluaciones inadecuadas de los criterios ambientales a la hora de evaluar los indicadores, Perevochtchikova (2013) plantea que es necesario considerar las limitaciones siguientes:

1. ¿Qué es lo que se pretende medir? Se hace necesario buscar qué pregunta se quiere responder.

2. Concertación de medidas a nivel multidisciplinario. Se debe tomar en cuenta que la gestión de los recursos naturales requiere, por su complejidad, un enfoque multidisciplinario e integrado.

3. Calidad de las mediciones. Se refiere a que se requiere certeza en la calidad de las mediciones de los elementos a estudiar (Perevochtchikova, 2013).

Se han realizado estudios relacionados con la gestión ambiental en diversos contextos (Arriaza Oliveros, 2010; García Céspedes, 2013; Ochoa Ávila, 2014; Zúñiga Igarza, 2011) para satisfacer las expectativas de la sociedad en cuanto a desarrollo sostenible, transparencia y responsabilidad, a fin de contribuir al "pilar ambiental" de la sostenibilidad (ONN, 2015) 


\section{Cuba y su gestión ambiental}

En Cuba, tanto la Constitución de la República (ANPP, 2019) como la Ley 81 del Medio Ambiente (ANPP, 1997) instituyen que el Estado protege el medio ambiente y los recursos naturales del país. La propia ley establece que el Ministerio de Ciencia, Tecnología y Medio Ambiente (CITMA), es el encargado de proponer la política ambiental y dirigir su ejecución, así como, en coordinación con otros órganos y organismos competentes, controlar y perfeccionar sistemáticamente la Estrategia Ambiental Nacional (EAN).

La EAN se traza para llevar a cabo la política ambiental y ha transitado por diferentes ciclos estratégicos. De acuerdo a la EAN 2016-2020 los principales problemas ambientales de Cuba son: degradación de los suelos; afectaciones a la cobertura forestal; contaminación; pérdida de la diversidad biológica; carencia y dificultades con el manejo, la disponibilidad y calidad del agua; impactos del cambio climático; deterioro de la condición higiénica sanitaria en los asentamientos humanos. Igualmente establece, dentro de las direcciones estratégicas, la mejora de la calidad ambiental y el perfeccionamiento de los instrumentos de la política y la gestión ambiental, y dentro de las Metas al 2020, el monitoreo de la contaminación (CITMA, 2016). Tomando en consideración estos elementos, las organizaciones adecuan sus estrategias a las del sector y alineadas a la EAN.

El proyecto social cubano valora como decisivos para la sostenibilidad y prosperidad de la nación, el uso racional y la protección de los recursos y el medio ambiente (PCC, 2017). Todo lo cual está contenido en los documentos aprobados en junio de 2017 y contienen la caracterización conceptual del proyecto, el instrumento de proyección a mediano y largo plazos, que facilitará la conducción estratégica de la economía nacional y la concreción de los objetivos.

Los documentos programáticos del proyecto social cubano reconocen la necesidad de promover el cuidado del medio ambiente, el fortalecimiento de la gestión de la información, así como la importancia de los indicadores para evaluar el cumplimiento de los objetivos y las metas ambientales (PCC, 2017), y a su vez están en concordancia con la EAN y los ODS.

Las organizaciones de todo tipo establecen su política ambiental de acuerdo a sus particularidades. La literatura reconoce aspectos generales tales como: debe ser redactada por la Dirección, reflejar la misión, visión y valores, demostrar compromiso y liderazgo, así como la Dirección debe asegurarse de que la política sea implementada y revisada periódicamente. Es importante considerar que todo lo que se exprese en la política es auditable y por lo tanto debería demostrarse mediante evidencias. Además, todos los planes, programas y proyectos de desarrollo económico y social deberán elaborarse o adecuarse en concordancia con los principios rectores de la política ambiental establecida por las autoridades competentes y a las disposiciones que procedan de estas (CITMA, 2016).

También, la norma NC-ISO 14001 del 2015 define que la alta dirección debe establecer, implementar y mantener una política ambiental dentro del alcance definido de su sistema de gestión ambiental, que:

- sea apropiada al propósito y contexto de la organización, incluida la naturaleza, magnitud e impactos ambientales de sus actividades, productos y servicios;

- proporcione un marco de referencia para el establecimiento de los objetivos ambientales;

- incluya un compromiso para la protección del medio ambiente, incluida la prevención de la contaminación, y otros compromisos específicos pertinentes al contexto de la 
organización;

- incluya un compromiso de cumplir con los requisitos legales y otros requisitos;

- incluya un compromiso de mejora continua del sistema de gestión ambiental para la mejora del desempeño ambiental.

La política ambiental debe:

- mantenerse como información documentada;

- comunicarse dentro de la organización;

- estar disponible para las partes interesadas (ONN, 2015)

Es por ello que debe valorarse si la política ambiental definida por la organización cumple con lo estipulado en las normas y en los documentos anteriormente mencionados.

Asimismo, esta norma NC 14001:2015 define que el desempeño ambiental está relacionado con la gestión de los aspectos ambientales y, en el contexto de un sistema de gestión ambiental, los resultados se pueden medir con respecto a la política ambiental de la organización, sus objetivos ambientales u otros criterios, mediante el uso de indicadores.

Además, explica que la organización debe determinar qué elementos requieren seguimiento y medición; los métodos de seguimiento, medición, análisis y evaluación, según corresponda, para asegurar resultados válidos; así como los criterios con los cuales la organización evaluará su desempeño ambiental y los indicadores apropiados. La propia norma aclara que se seleccionan indicadores para evaluar el logro de los objetivos ambientales medibles y que es posible usar métodos cuantitativos o cualitativos con relación a una escala especificada para determinar si se ha logrado el objetivo ambiental. En el presente trabajo se justifica la razón para determinar los indicadores seleccionados y se crea una escala.

\section{La gestión ambiental en las organizaciones cubanas}

Considerando que las organizaciones inciden en el estado del medio ambiente y tienen variadas estructuras y subordinaciones, en la EAN 2016-2020 se identifican características del panorama nacional, tales como: el perfeccionamiento de las estructuras y funciones de los Organismos de la Administración Central del Estado (OACE) y otras entidades nacionales, fundamentalmente por separación de las funciones estatales de las empresariales; el fortalecimiento de las estructuras de organización de la sociedad civil y voluntad para incluir el tema de la protección del medio ambiente en sus agendas de trabajo; el impacto de la actividad de las nuevas formas de gestión económica sobre el medio ambiente y; el fortalecimiento del papel de los territorios y la gestión local (CITMA, 2016).

En el propio documento se plantea que CITMA, en su condición de OACE rector de la política ambiental, es el encargado de proponerla y controlar su implementación" así como, todos los OACE y entidades bajo cualquier modelo de gestión tienen la obligación de incorporar aspectos relacionados con la protección ambiental, en correspondencia con las políticas públicas establecidas.

EI CITMA, cumpliendo su función de control visita a las organizaciones para verificar aspectos relacionados con la política ambiental, entre los que se encuentran: el diagnóstico ambiental, las inversiones para la solución de los problemas ambientales, las actividades de divulgación y capacitación ambiental de sus recursos humanos, entre otros. 
Es importante considerar el cumplimiento de las regulaciones ambientales y sanitarias aplicables a las entidades, que son verificadas por las inspecciones estatales de los organismos rectores Ministerio de Salud Pública (MINSAP), Ministerio de la Industria Alimentaria (MINAL), Instituto de Nacional de Recursos Hídricos (INRH), Ministerio del Interior (MININT) y CITMA (CITMA, 2011).

Asimismo, existen otras partes interesadas como el Grupo de Trabajo Estatal de la Bahía de La Habana (GTE BH) que promueve la gestión ambiental y actúa directamente con las organizaciones que tienen alguna incidencia en la bahía de La Habana. El GTE BH tiene entre sus funciones: controlar el cumplimiento de los planes de medidas y soluciones ambientales previstas en el plan de medidas a corto, mediano y largo plazo para mitigar los impactos ambientales y controlar el cumplimiento del "Programa de enfrentamiento a la contaminación de las bahías de interés nacional". Además, como entidad referente de los procesos de gestión, elaboró indicadores que permiten comparar el desempeño ambiental pasado y presente de una organización para que fueran empleados de manera voluntaria por las empresas bajo su jurisdicción de control.

Además, los OACE y cada Organización Superior de Dirección Empresarial (OSDE) subordinada a estos, inspeccionan la correcta implementación de la política ambiental.

Las entidades que realizan las visitas consideran un conjunto de elementos a evaluar y que deben tenerse en cuenta para la evaluación del desempeño ambiental a lo interno de la organización. Dado que los indicadores son una herramienta muy útil para el seguimiento y control, se hace necesario diseñar indicadores que estén en concordancia con estos elementos.

\section{Desempeño ambiental}

Se han desarrollado plataformas, iniciativas, metodologías para que las organizaciones y los países puedan mostrar su desempeño ambiental, tales como: el ranking entre países que crea el Índice de Desempeño Ambiental (EPI) al utilizar métodos rigurosos y ofrecer una visión holística de la protección del medio ambiente. (Oregi \& Galera, 2013), los informes de sostenibilidad GRI (GRI, 2017), la iniciativa EPI Euskadi 2013 a escala regional (Oregi \& Galera, 2013) y en diferentes organizaciones (López, 2010; Cira, 2004; Leiva Mas, 2011; Medel González et al., 2015; Bovea et al., 2017; Air Products and Chemicals, 2018; FUCAC, 2018; Iberdrola, 2018; Siemens Gamesa, 2018; Stantec, 2017; TOYOTA, 2018)

Para diagnosticar el desempeño ambiental de una organización se pueden utilizar disimiles metodologías, procedimientos y formas de hacer, pero es necesario analizar el contexto y si son adecuadas. Los informes de sostenibilidad abarcan aspectos que en el contexto cubano son logros cotidianos, como los indicadores relacionados con las prácticas laborales y los derechos de los trabajadores, que forman parte del quehacer laboral a nivel nacional, así como los relacionados con los derechos humanos, sociedad y responsabilidad, por lo que no son aplicables. En la bibliografía consultada no se encontraron referentes a organizaciones que cuantifiquen su desempeño ambiental y que impactan sobre bahías priorizadas.

En Cuba, se utiliza de manera sistemática, la Metodología para la ejecución de los diagnósticos ambientales para la obtención del reconocimiento ambiental nacional, diseñada por el CITMA e implementada desde el 2012. Las organizaciones se auxilian de ella para postularse como candidatas a la obtención del Reconocimiento Ambiental Nacional (RAN), que es una de las formas de premiar la excelencia en materia de gestión ambiental y la información que brindan incluye el año en curso (CITMA, 2011). Esta metodología es abarcadora y tiene en cuenta los siguientes aspectos: responsabilidad de la dirección de la entidad con la solución 
de los problemas ambientales; cumplimiento de la legislación ambiental; minimización de la contaminación; uso eficiente de los recursos naturales; residuos y emisiones; productos químicos y desechos peligrosos; gestión ambiental y procesos productivos. Sin embargo, no considera los criterios de los partes interesados ni establece niveles de desempeño ambiental.

La EAN 2016 - 2020 establece objetivos y acciones prioritarias, entre ellos: Disminuir la contaminación como vía para mejorar la calidad ambiental, implementación del Programa de erradicación de fuentes contaminantes que afectan fuentes de abasto de agua $2014-2020$ y el Programa de enfrentamiento a la contaminación de las bahías.

En este artículo se analizó una organización cubana que incide en una bahía, por lo que la evaluación de su desempeño ambiental contribuye a dar cumplimiento al citado objetivo y en consonancia con estos programas. También se propicia el cumplimiento a las metas nacionales de diversidad biológica que ha adoptado el país de acuerdo con el Programa Nacional sobre la Diversidad Biológica (PNDB) 2016- 2020, en particular la Meta nacional 6: Se reduce la contaminación ambiental a límites ecológicamente seguros para la salud humana, animal y vegetal (CITMA, 2015). La meta nacional se relaciona directamente con la Meta 8 del Plan Estratégico para la Diversidad Biológica 2011-20204 (CDB, 2010) y también tributa a la Agenda al 2030 para el Desarrollo Sostenible, con énfasis en el ODS 6: Garantizar la disponibilidad y la gestión sostenible del agua y el saneamiento para todos, con sus meta 6.3: De aquí a 2030, mejorar la calidad del agua reduciendo la contaminación, eliminando el vertimiento y minimizando la emisión de productos químicos y materiales peligrosos, reduciendo a la mitad el porcentaje de aguas residuales sin tratar y aumentando considerablemente el reciclado y la reutilización sin riesgos a nivel mundial (ONU, 2016).

El desempeño ambiental de una organización no puede decidirse unilateralmente, es necesario tener argumentos que puedan contrastarse y combinarse, que los criterios tengan suficientemente definidas las pautas, para poder establecer comparaciones y sean suficientemente flexibles para ser aplicables en varios contextos; por lo se elige emplear el método Delphi. Este es un método estructurado e iterativo que utiliza cuestionarios por rondas para que los expertos den respuesta sobre una determinada problemática y en forma estadística se construye un acuerdo consensuado de valor agregado. El método Delphi combina lo mejor de cada experto con las mejores prácticas del trabajo en grupo. Por sus características de anonimato no hay presiones ni influencias, se modifican las opiniones de manera espontánea si en la retroalimentación se encuentran argumentos más apropiados y se obtienen las mejores alternativas basadas en la inteligencia y el aporte colectivo.

Teniendo en cuenta estos aspectos y por la necesidad de dar seguimiento a las acciones ambientales que se realizan, el objetivo de este trabajo es contribuir a la mejora de la evaluación del desempeño ambiental en una organización cubana.

\footnotetext{
${ }^{4}$ Las Partes en el Convenio sobre la Diversidad Biológica, en 2010 en Nagoya, Japón, aprobaron el Plan Estratégico de la Diversidad Biológica 2011-2020, con el propósito de inspirar acciones a gran escala por todos los paises y las partes interesadas en apoyar la diversidad biológica. Conocida como Metas Aichi.
} 


\section{MATERIALES Y MÉTODOS}

Para identificar los aspectos relacionados con la evaluación del desempeño ambiental que se llevan a cabo en la entidad, se analizó el estado actual, lo que posibilitó detectar insuficiencias que podrían atentar contra una adecuada toma de decisiones. Al realizar el diagnóstico del comportamiento ambiental de la organización, se definieron tres pasos metodológicos, los cuales se pueden observar en la Figura 1.

\section{Analizar política ambiental}

- Verificar si está diseñada acorde a la literatura

- Verificar si se corresponden con la lista de chequeo.

-Si existe relación entre la estrategia ambiental nacional, la estrategia sectorial y la propia.

- Identificar los objetivos y metas ambientales declarados.

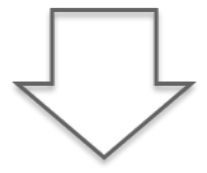

\section{Realizar diagnóstico ambiental}

- Aplicar la lista de chequeo basada en la Metodología para la ejecución de los diagnósticos ambientales para la obtención del reconocimiento ambiental nacional (RAN).

-Si se ha realizado previamente, hacer una evaluación comparativa. Si fue en un periodo menor de 1 año, solo valorarlo.

- Derminar los indicadores ambientales identificados en la organización.

- Determinar indicadores ambientales que se informan (ONEI, para los ODS y otros)

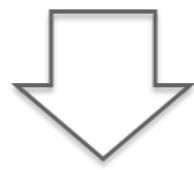

\section{Analizar criterios de desempeño ambiental}

- Identificar los criterios de desempeño ambiental de las partes interesadas

- Verificar que existan indicadores que permitan dar seguimiento al cumplimiento de los criterios de desempeño ambiental

- Utilizar una ficha técnica que contenga los datos de los indicadores

- Clasificar los indicadores de acuerdo a las categorías: "indicadores de eficiencia", "indicadores básicos de gestión" e "indicadores básicos de legislación"

-Evaluar los indicadores por categorias y por nivel de cumplimiento

Figura 1. Pasos metodológicos para el diagnóstico del comportamiento ambiental de la organización. Elaboración propia.

Paso 1. En el primer paso se analizó la política ambiental de acuerdo con los referentes internacionales y al contexto cubano.

Paso 2. Dada la integralidad de la metodología de diagnóstico para alcanzar el RAN, se 
efectuó el diagnóstico ambiental utilizando una lista de chequeo basada en dicha metodología y se realizaron entrevistas a directivos, trabajadores del centro y partes interesadas; así como la revisión de documentos, compilación de información y la observación directa.

Se utilizó la lista de chequeo porque se considera la primera evidencia del estado actual de la organización, que debe ir acompañada de otros análisis como se muestra en la figura 1. Estos análisis tienen su fundamento en las normas internacionales de gestión y de desempeño ambiental y en las bases en que se sustentan la política ambiental nacional.

Se revisó el informe de diagnóstico ambiental ya que estaba actualizado (menos de un año de realizado).

Se aplicó la metodología para obtener el RAN, se explicitaron los aspectos más importantes que están presentes en el área de influencia de la entidad y dentro de la propia entidad, que tienen incidencia significativa en el desempeño ambiental. Es así que se consideraron los aspectos económicos, el uso de los portadores energéticos, las condiciones higiénicosanitarias, el empleo de productos químicos, la generación de residuos, entre muchos otros elementos. A estos aspectos se asociaron indicadores tales como el cumplimiento del plan técnico-económico y de los índices de consumo; el manejo de los productos químicos y de los residuos recuperables o reutilizables, la disponibilidad de medios de protección y el establecimiento de metas ambientales.

Estos indicadores son válidos para cualquier organización y se adecuaron a las características propias. Asimismo, tomando en consideración los objetivos y metas ambientales que fueron declarados y que se identificaron en el paso anterior; se verificó que existían indicadores que posibilitaban darle seguimiento.

Es decir, en este paso se analizaron los indicadores ambientales que ya habían sido identificados por la organización: los que se tuvieron en cuenta cuando se aplicó la lista de chequeo basada en la metodología para el RAN, los que se incluyeron en la política ambiental y los que habían incorporado de los propuestos por el GTE BH. Se identificaron cuáles de estos son informados y el programa ambiental que se habían propuesto.

En el paso tres, se determinó que las partes interesadas para esta organización eran el CITMA, el GTE BH y la OSDE a la que está subordinada. Los criterios fundamentales de desempeño ambiental asociados a estos, fueron: la política ambiental, el diagnóstico ambiental, las inversiones para la solución de los problemas ambientales, las actividades de divulgación y capacitación ambiental de sus recursos humanos, entre otros.

Para dar cumplimiento a los criterios de desempeño ambiental se analizaron los indicadores existentes y se detectó la falta de otros, por lo que se crearon nuevos indicadores.

Se elaboró una ficha técnica que registre los datos de todos los indicadores.

Se crearon categorías que agruparon los indicadores para delimitar sobre que esferas dirigir los esfuerzos. En estas se encontraban los indicadores de eficiencia, básicos de gestión y de legislación.

Se aplicó el Método Delphi a cinco especialistas considerados como expertos, dos pertenecientes a la organización y tres externos. En la selección inicial de ocho especialistas se tuvieron en cuenta criterios de experiencia, conocimientos, posición, responsabilidad, acceso a la información y disponibilidad. Se eligieron cinco especialistas porque obtuvieron un índice de experticia alto (mayor que 0,7); más de quince años de experiencia en la actividad; todos tenían categoría científica; cuatro poseían categoría docente superior; todos tenían publicaciones relacionadas con el tema en revistas referenciadas y todos habían realizado cursos de postgrado vinculados con la temática, en el último año. Además, todos 
habían sido considerados como expertos con anterioridad en al menos seis oportunidades.

Por estos motivos y ser reconocidos como profesionales prestigiosos, fueron seleccionados como expertos. Los expertos seleccionados ocupan cargos directivos (dos), de control (dos) y uno en la propia actividad, de tal manera que las visiones de la evaluación consideraron la planificación, el control y el benchmarking.

Los especialistas concordaron en que los pasos metodológicos para el diagnóstico del comportamiento ambiental de la organización que se implementaron eran apropiados y que los nuevos indicadores eran adecuados.

\section{RESULTADOS}

Una vez llevado a cabo el análisis de la política se verificó que no contiene todos los elementos acorde a la literatura. Se puede señalar que desde la alta dirección existe una declaración de principios y objetivos. A grandes rasgos, las líneas de actuación están definidas, pero no se especifican las responsabilidades de manera explícita.

Los ítems declarados en la política ambiental no siempre se corresponden con la lista de chequeo.

Existe una correspondencia plena entre la estrategia de la organización, la estrategia del OSDE al que se subordina y con lo dictaminado por su Ministerio, acorde a lo dispuesto en la Ley 81. De igual manera, la política y los programas establecidos están diseñados en aras de gestionar de manera responsable los recursos asociados a su objeto social y sobre todo, a minimizar el impacto sobre el ecosistema de la Bahía de La Habana.

En cuanto a los objetivos y metas declarados en la organización, se realizan las observaciones siguientes:

- no todas las metas tienen establecido un período de cumplimiento,

- no todos los objetivos tienen una meta asociada,

- todos los objetivos y metas no tienen un indicador asociado,

- no en todos los casos existe una clara definición de los objetivos y las metas,

- en algunos casos se definen como metas lo que debían ser indicadores y viceversa.

Tomando en consideración los aspectos ambientales que son inspeccionados en las visitas que realizan los especialistas de las partes interesadas se exponen a continuación los Resultados del diagnóstico del comportamiento ambiental de la organización.

Los aspectos evaluados como positivos son los siguientes:

- existencia de las evidencias documentadas acerca del compromiso de la alta dirección de la entidad con la solución de los problemas ambientales. Estas son: las actas de los consejos de dirección, de las reuniones de trabajo de las inversiones para minimizar los impactos al medio ambiente, los planes de medidas para su solución y las actas de las reuniones que se realizan para su seguimiento y control; 
- aplicación del Reglamento Higiénico Sanitario y Ambiental. El cumplimiento estricto se verifica mediante inspecciones y en caso de violaciones, se notifica a los infractores y se toman medidas disciplinarias a los trabajadores y directivos responsables;

- medición mensual de indicadores que evalúan la eficacia de la gestión ambiental;

- $\quad$ se llevan a cabo acciones que minimizan el impacto negativo sobre el ecosistema de la Bahía de La Habana, tales como:

- vigilancia trimestral a la composición de las aguas residuales (mediante análisis con los laboratorios competentes, para verificar el cumplimiento y eficacia de las acciones tomadas);

- empleo de productos biodegradables para la limpieza y desinfección de las áreas productivas;

- mantener y revisar periódicamente las acciones del Plan de Producción más Limpia;

- asegurar el uso racional del agua en los procesos productivos;

- uso de pistolas de agua para regular el consumo en los salones de proceso durante la jornada laboral y en la limpieza;

- sistematicidad en la recogida de los sólidos;

- chequeo continuo del cumplimiento de la legislación vigente;

- realización de capacitación sistemática a todo el personal en la temática ambiental creando paulatinamente una mentalidad ambientalista;

- considerar los criterios ambientales en la política de compras y en las prácticas de almacenamiento;

- colocación de chimeneas ecológicas que minimizan las concentraciones de gases nocivos y monitorear su funcionalidad;

- seguimiento a las acciones para el manejo de los productos químicos y desechos peligrosos;

- implementación de un sistema de gestión ambiental;

- existencia del Sistema de Gestión de la Calidad certificado;

- implementación del Plan de Mantenimiento industrial.

\section{Diagnóstico ambiental}

Tomando en consideración que la especialista ambiental había efectuado un diagnóstico ambiental en la organización, según la metodología para la ejecución de los diagnósticos ambientales, seis (6) meses atrás, se realizó una valoración de este. Del análisis se concluyó que a pesar de que se elaboró de acuerdo a la Metodología RAN no fueron tenidos en cuenta aspectos importantes tales como: el uso de sustancias agotadora de la capa de ozono (SAO), la consideración de los criterios ambientales en la política de compras y en las prácticas de almacenamiento, áreas verdes, jardinería y áreas exteriores; y la definición de los elementos componentes del sistema de gestión ambiental (política, objetivos y metas ambientales, así como el programa de acción). 


\section{Identificación de indicadores ambientales en la organización}

La organización cuenta con un conjunto de indicadores que dan respuesta a los aspectos contenidos en la metodología RAN, pero no son insuficientes los relacionados con los objetivos y metas ambientales.

En coordinación con el GTE BH, la organización tiene identificados e implementados los siguientes indicadores que son calculados anualmente:

1. Porcentaje de objetivos y metas ambientales alcanzadas.

2. Carga contaminante emitida.

3. Carga contaminante emitida por tonelada de producción.

4. Porcentaje de residuos reutilizados.

5. Cantidad de energía consumida por tonelada de producción.

6. Consumo de agua por tonelada de producción.

7. Porcentaje de inversiones destinadas a la solución de problemas ambientales.

8. Recursos humanos capacitados en temáticas ambientales.

De acuerdo a cada indicador, existen áreas encargadas de proporcionar la información necesaria. El Departamento de Economía factura mensualmente las ventas de los desechos sólidos a entidades de producción porcina; la electricidad y el consumo de agua se controlan mensualmente por el área Energética y se emiten partes de cumplimiento de esos indicadores. El caudal y la carga contaminante de los residuales líquidos son determinados anualmente por entidades externas especializadas y se puede solicitar monitoreo intermedio ante cambios de materias primas, flujo producción, y otros.

Los indicadores que dan cumplimiento a los ODS y otros indicadores ambientales seleccionados, se informan a nivel de OACE. Por ser una entidad subordinada a un grupo empresarial no existen indicadores ambientales de los que se publican en las estadísticas oficiales que se informan directamente a la ONEI ni para los ODS, aunque esta información se inserta en los flujos inter organizacionales. Por la propia naturaleza de la entidad existen un conjunto de indicadores ambientales que están relacionados con los ODS y otras informaciones, que conforman los datos que el sector reporta a las instancias competentes.

A partir del diagnóstico y conforme a la bibliografía consultada, la política ambiental se perfeccionó, así como los objetivos y metas. En función de estos objetivos y metas se elaboró el Programa Ambiental de acuerdo al formato que se muestra en la Tabla 3.

Fueron calculados los indicadores que contaban con la información necesaria y se propuso que este cálculo se realizara dos veces al año. 


\section{DISCUSIÓN}

La lista de chequeo es la primera aproximación que realiza la organización como autodiagnóstico en la entidad. A pesar de la amplia gama de elementos que son considerados en la metodología RAN, estos no se integran, no existe clasificación de los indicadores ni se ponderan: razón por la cual la metodología que se propuso contribuye a complementarla.

Las evaluaciones de desempeño ambiental se realizan anualmente, sin embargo, para poder hacer las correcciones pertinentes en un período de tiempo más corto, los cálculos de los indicadores deben realizarse semestralmente.

A partir de la valoración realizada, el diseño de los nuevos indicadores tiene en cuenta los siguientes aspectos:

1. Objetivos y metas.

2. Legislación.

3. Portadores energéticos (agua y electricidad).

4. Contaminación (residuos sólidos, residuos líquidos, residuos peligrosos, los productos químicos peligros y el drenaje pluvial).

5. Aplicación de la ciencia y la innovación tecnológica.

6. Formación ambiental.

7. Inversiones destinadas a la protección del medio ambiente.

8. Implementación y cumplimiento de los planes de acción.

Las organizaciones no solo deben conocer su desempeño ambiental, también deben evaluarlo para identificar avances y retrocesos en su relación con el medio ambiente (Medel González et al., 2015). En tal sentido se diseñaron indicadores complementarios a los ya existentes para evaluar otros aspectos. Con vistas a obtener una valoración más precisa del nivel de cumplimiento, a todos los indicadores se les otorga puntuación, desglosados en "parcial", "en proceso" y "total", de forma tal que se puedan comparar periodos "en avance" o no.

Con la misma intencionalidad los indicadores se agrupan de acuerdo a las categorías siguientes:

- Indicadores de eficiencia. Relacionados con el objeto social de la organización en armonía con el medio ambiente y con los indicadores básicos de contaminación (manejo de productos químico y desechos peligrosos, tratamiento de las emisiones a la atmósfera, ruidos y vibraciones). El total de puntos a alcanzar es 20.

- Indicadores básicos de gestión. Son los que debe implementar cada organización y el valor máximo es de 30 puntos. Incluye entre otros aspectos: diagnóstico ambiental, política ambiental, programa de gestión ambiental. 
- Indicadores básicos de legislación. Contienen el marco legal básico específico de la entidad y se puede obtener como máximo un total de 50 puntos.

El total de puntos es 100 y la mitad corresponde a los indicadores básicos de legislación por lo importante de su cumplimiento. De manera independiente se podría considerar el nivel de desempeño ambiental de acuerdo a la Tabla1.

Tabla 1. Nivel de desempeño ambiental.

\begin{tabular}{|c|c|c|}
\hline Rango Total & Legislación & Estado \\
\hline De 90 a 100 & $39-50$ & Avanza \\
\hline De 89 a 69 & $38-27$ & Se estanca \\
\hline$<0=68$ & $<27$ & En retroceso \\
\hline
\end{tabular}

Al efectuar el cálculo de los indicadores ambientales se apreció que aunque en la organización se realizan acciones para mejorar su desempeño, se incumplen normas importantes, por lo que la puntuación no es favorable en el aspecto "legislación" y de acuerdo a la Tabla 1, el estado del nivel de desempeño fue "se estanca". Sin embargo, esta situación debe ir mejorando con la construcción de una planta de tratamiento de residuales que se edifica como parte de un proyecto inversionista.

En la literatura consultada aparecen investigaciones relacionadas con el medio ambiente y el empleo de indicadores; entre las que se encuentran: las que integran las normas ISO (Bravo Avalos, 2017; Cira, 2004; Oliveira et al., 2016; Pérez García, 2013; Ulloa Enríquez, 2012; Cira et al., 2012) y desde la estrategia empresarial (Corella, 2013), pero no creando los niveles: Avanza, Se estanca y En retroceso.

Los estudios relacionados con el desempeño ambiental se aplican a destinos turísticos (López Moreda, 2010), en instituciones de educación superior (Cira, 2004; Leiva Mas, 2011), en centrales eléctricas de la generación distribuida (Medel González et al., 2015) y la metodología de Análisis de Ciclo de Vida para evaluar el desempeño ambiental de los sistemas de gestión de residuos sólidos (Bovea et al., 2017), pero no incluyen otros aspectos de la política ambiental, ni propician la evaluación de estadios cuantitativos.

Muchas organizaciones publican sus informes de sostenibilidad, que incluye su incidencia en la sociedad y la economía que son más exhaustivos, pero valora, por ejemplo: aspectos como la proporción de mujeres empleadas, el porcentaje de mujeres en cargos directivos; que son conquistas del proyecto social cubano. Muestras de ello son los informes de lberdrola SA, Siemens Gamesa, FUCAC, Air Product, Stantec, Toyota, Carburos Metálicos, entre otros (Air Products and Chemicals, 2018; FUCAC, 2018; Iberdrola, 2018; Siemens Gamesa, 2018; Stantec, 2017; TOYOTA, 2018; GRUPO ÉXITO, 2018).

La ficha es un elemento esencial en la construcción de indicadores por lo que se propone que la ficha de la ONEI se utilice para todos los indicadores con la finalidad de estandarizar formatos y a que esté disponible toda la información necesaria para construir indicadores. En la Tabla 2 se muestran los componentes de la ficha. 
Tabla 2. Ficha técnica de los indicadores.

\begin{tabular}{|c|c|c|c|c|c|}
\hline \multicolumn{6}{|c|}{ Identificación de la variable/indicador } \\
\hline \multicolumn{6}{|c|}{ 1. Nombre del indicador: } \\
\hline \multicolumn{6}{|c|}{ 2. Unidad de medida del indicador: } \\
\hline \multicolumn{6}{|c|}{ 3. Periodicidad de los datos: } \\
\hline \multicolumn{6}{|c|}{ 4. Cobertura } \\
\hline \multicolumn{6}{|c|}{ 5. Periodo de la serie que actualmente está disponible: } \\
\hline \multicolumn{6}{|c|}{ Descripción general del Indicador } \\
\hline \multicolumn{6}{|c|}{ 1. Definición del indicador: } \\
\hline \multicolumn{6}{|c|}{ 2. Fórmula del indicador: } \\
\hline \multicolumn{6}{|c|}{ 3. Definición de las variables: } \\
\hline \multicolumn{6}{|c|}{ 4. Fuente de datos: } \\
\hline \multicolumn{6}{|c|}{ 5. Disponibilidad de los datos: } \\
\hline Variable & $\begin{array}{l}\text { Fuente primaria } \\
\text { de la información: }\end{array}$ & Periodicidad & $\begin{array}{l}\text { Periodo de la } \\
\text { serie disponible }\end{array}$ & $\begin{array}{l}\text { Disponibilidad } \\
\text { de la variable }\end{array}$ & $\begin{array}{l}\text { Forma } \\
\text { presentación } \\
\text { de los datos }\end{array}$ \\
\hline Desde & Hasta & $\begin{array}{l}\text { Plenamente } \\
\text { disponible }\end{array}$ & Digital & Impreso & \\
\hline \multicolumn{6}{|c|}{ 6. Limitaciones del indicador: } \\
\hline \multicolumn{6}{|c|}{ 7. Entidades que intervienen en la elaboración del indicador y forma en que interviene: } \\
\hline \multicolumn{6}{|c|}{ Relevancia o pertinencia del indicador } \\
\hline \multicolumn{6}{|c|}{ 1. Finalidad/propósito/importancia del Indicador: 2. Tema: } \\
\hline \multicolumn{6}{|c|}{3 Objetivo / meta de política: } \\
\hline \multicolumn{6}{|c|}{ 4. Relación con la sostenibilidad: } \\
\hline \multicolumn{6}{|c|}{ 5. Convenios y acuerdos Internacionales con los que se relaciona el indicador: } \\
\hline \multicolumn{6}{|c|}{ 6. Propuesta de representación con análisis de tendencia } \\
\hline \multicolumn{6}{|c|}{ 7. Observaciones: } \\
\hline \multicolumn{6}{|c|}{ 8. Bibliografía } \\
\hline 9. Forma & vantamiento de la in & formación & & & \\
\hline
\end{tabular}

Fuente: ONEI (2014)

El programa ambiental posibilita un seguimiento más efectivo, puesto que se establecen periodos de cumplimiento y sus responsables, por tal motivo, mientas más detallado sea, será más fácil para el tomador de decisiones incidir sobre aquellos aspectos que tengan dificultades y sobre las personas que deben ejecutarlas. Para el Programa Ambiental se adoptó el formato que se muestra en la Tabla 3. 
Tabla 3. Formato del Programa Ambiental

\begin{tabular}{|c|c|c|c|c|c|c|c|}
\hline \multirow{2}{*}{ Acción } & \multicolumn{2}{|c|}{$\begin{array}{c}\text { Fecha } \\
\text { cumplimiento }\end{array}$} & \multirow{2}{*}{ Responsable } & \multirow{2}{*}{ Ejecutan } & \multirow{2}{*}{$\begin{array}{l}\text { Estado de } \\
\text { ejecución }\end{array}$} & \multirow{2}{*}{$\begin{array}{l}\text { Actualizado } \\
\text { por: }\end{array}$} & \multirow{2}{*}{ Observaciones } \\
\hline & $\frac{\frac{\pi}{0}}{\alpha}$ & $\begin{array}{l}\overline{\mathbb{N}} \\
\propto \\
\propto\end{array}$ & & & & & \\
\hline
\end{tabular}

\section{CONCLUSIONES}

Se diseñaron los Pasos metodológicos para el diagnóstico del comportamiento ambiental de la organización que incluyó el diagnóstico inicial, la identificación de los indicadores ambientales que están implementados y otros criterios de evaluación de desempeño ambiental a ser considerados.

Se detectaron deficiencias en: la política ambiental declarada por la organización, los objetivos, metas e indicadores.

Se realizaron modificaciones a los criterios de evaluación de desempeño ambiental, a la política ambiental declarada por la organización, así como a los objetivos y metas.

Se diseñaron nuevos indicadores para dar seguimiento y control, que se calcularon de acuerdo con la información disponible.

El empleo de indicadores de evaluación del desempeño ambiental permitió a la organización delimitar sus aciertos y desaciertos e incidir sobre ellos, así como cuantificar de manera resumida los resultados de su política ambiental, estableciendo cuál era su nivel de desempeño ambiental y con ello, adecuar su accionar; lo que contribuyó a la mejora de su evaluación del desempeño ambiental.

\section{REFERENCIAS}

AIR PRODUCTS AND CHEMICALS, Inc. (2018). Informe de Sostenibilidad 2018 (Extracto UE) [en línea].[Consulta 09 abril 2019]. Disponible en: https://www.carburos.com/ /media/ Files/PDF/company/2018-sustainability-report-es.pdf.

ARGENTINA. CONSEJO NACIONAL DE COORDINACIÓN DE POLÍTICAS SOCIALES. (2018). Objetivos de desarrollo sostenible. Informe país ARGENTINA 2018. [en línea]. 
Biblioteca del Congreso: Argentina. [Consulta 09 abril 2019] Disponible en: http://www. odsargentina.gob.ar/public/documentos/seccion_publicaciones/resumen_informe_ pais_2018_espanol_web.pdf

ARRIAZA OLIVEROS, L. (2010). Metodología para la estimación de corrientes marinas como herramienta en la gestión ambiental de la zona marina costera de Cuba [disco]. Tesis Doctoral. Instituto Superior de Tecnologías y Ciencias Aplicadas. La Habana.

Asamblea Nacional del Poder Popular. ANPP. Ley No. 81. Del Medio Ambiente. (1981). Gaceta Oficial de la República de Cuba [en línea]. La Habana. Cuba. [Consulta 09 abril 2019]. Disponible en: http://www.oas.org/dsd/fida/laws/legislation/cuba/cuba_81-97.pdf

Asamblea Nacional del Poder Popular. ANPP. Constitución de la República de Cuba (2019) Gaceta Oficial de la República de Cuba GOC-2019-406-EX5 [en línea]. La Habana. Cuba. [Consulta 25 abril 2019]. Disponible en: http://www.gacetaoficial.gob.cu/

BOVEA, M. D, et al. (2010). Aplicación de la metodología de Análisis de Ciclo de Vida para evaluar el desempeño ambiental de sistemas de gestión de residuos en Iberoamérica. Revista Internacional de Contaminación Ambiental [en línea]. 32 (Especial Residuos Sólidos). 23-46. [Consulta 25 marzo 2019]. Disponible en: DOI: http://dx.doi.org/10.20937/RICA.2016.32.05.03

BRAVO AVALOS, M. B. (2017). Sistema integrado de gestión de calidad ambiental para la zona natural andina ecuatoriana de la Provincia de Chimborazo [disco]. Tesis Doctoral. Universidad de La Habana.

CITMA. (2011). Metodología para la ejecución de los diagnósticos ambientales, para la obtención del Reconocimiento Ambiental Nacional (RAN) [disco]. La Habana.

CITMA. Estrategia Ambiental Nacional 2016 / 2020 (2016) [en línea]. La Habana, Cuba. [Consulta 17 septiembre 2018]. Disponible en: http://repositorio.geotech.cu/jspui/ bitstream/1234/2727/1/Estrategia\%20Ambiental\%20Nacional\%202016-2020.pdf

CDB. Convenio de Diversidad Biológica. (2010). Plan Estratégico para la Diversidad Biológica 2011-2020. [Consulta 25 de enero de 2019]. Disponible en: www.cbd.int/sp

CORELLA, C. M. V. (2013). Contribución a la gestión estratégica organizacional con enfoque ambiental. Enl@ce: Revista Venezolana de Información, Tecnología y Conocimiento [en línea]. 10(1), 31-52. [Consulta 25 de enero 2019]. Disponible en: https://dialnet.unirioja.es/ servlet/articulo?codigo $=4334685$

CUBA. (2019) Informe nacional sobre la implementación de la agenda 2030 [en línea]. [Consulta 27 de agosto 2019]. Disponible en: https://foroalc2030.cepal.org/2019/sites/foro2019/files/ informe_nacional_voluntario_de_cuba_sobre_implementacion_de_la_agenda_2030.pdf

DECHKOVA, D.M. y MIRANDA, R. (2014). Company Sustainability Reporting: Decision Making Model Utilising Compensatory Fuzzy Logic. En: Espin R., Pérez R., Cobo A., Marx J., Valdés A. (eds). Soft Computing for Business Intelligence [en línea]. Springer. Berlin, Heidelberg. Disponible en: https://doi.org/10.1007/978-3-642-53737-0_17

ESPAÑA. (2018). Informe de España para el examen nacional voluntario 2018 [en línea]. 
España. [Consulta 09 abril 2019]. Disponible en: https://sustainabledevelopment.un.org/ content/documents/20113Spain_VNR_Report_Spain_29_de_junio_2018.pdf

FUCAC. (2018). Pertenecer: Informe de Sostenibilidad 2018 [en línea]. [Consulta 09 abril 2019]. Disponible en: http://deres.org.uy/wp-content/uploads/2018/11/Reporte-deSostenibilidad-2018.pdf

GAETANI, F. (2018). Actividades de ONU Medio Ambiente para fortalecer la capacidad de la región de producir datos e información ambiental de manera sostenida y sistemática [en línea]. Santiago. Chile. [Consulta 29 marzo 2019]. Disponible en: https://www.cepal.org/sites/ default/files/presentations/cea-ce17-onu-medio-ambiente-actividades-fortalecer-capacidadproducir-datos-ambientales-04-10-2018.pdf

GARCÍA CÉSPEDES, D. (2013). Metodología de gestión ambiental para agroecosistemas con probables riesgos a la salud por presencia de contaminación química [disco]. Tesis Doctoral. Instituto Superior de Tecnologías y Ciencias Aplicadas. La Habana.

GRI. GLOBAL REPORTING INITIATIVE. Guía para la elaboración de memorias de sostenibilidad [en línea] [Consulta 25 enero 2019] Disponible en: http://www.mas-business. com/docs/Spanish-G4.pdf

GRUPO ÉXITO. (2018). Innovación como palanca de diferenciación. Informe Integrado 2018 [en línea]. [Consulta 09 abril 2019]. Disponible en: https://www.grupoexito.com.co/ phocadownload/asambleas/2018/marzo/Informe-integrado-Grupo-Exito-2018.pdf

IBERDROLA. (2018). Estado de información no financiera. Informe de sostenibilidad Ejercicio 2018 [en línea]. [Consulta 29 marzo 2019]. Disponible en: https://www.iberdrola.com/wcorp/ gc/prod/es_ES/corporativos/docs/IB_Informe_Sostenibilidad.pdf

CIRA, I. G. (2004) Modelo de gestión integrada calidad-medioambiente (CYMA) aplicado en organizaciones cubanas [en línea]. Tesis Doctoral. Instituto Superior Politécnico "José Antonio Echeverría" (Cujae). [Consulta 25 enero 2017]. Disponible en: http://tesis.cujae.edu. $\mathrm{cu}$

CIRA, I. G., et al. (2010). Indicadores para la evaluación del desempeño ambiental de los Centros de Educación Superior (CES). Revista CENIC [en línea]. 41(4). [Consulta 25 marzo 2019]. Disponible en: https://revista.cnic.edu.cu/revistaCQ/sites/default/files/articulos/CQ2010-4-CQ-041.pdf

ISO-26000. (2010) Guía de responsabilidad social [en línea]. [Consulta 25 de enero de 2019]. Disponible en: http://www.iso.org/iso/home.

JUNTA DE ANDALUCÍA. (2014). Los indicadores ambientales: definición, evolución y aplicaciones [en línea]. [Consulta 8 de mayo de 2018]. Disponible en: http://clima.ue.org/ documentos/climaSIG.htm.

LEIVA MAS, J. Evaluación de soluciones tecnológicas en la Universidad Central "Marta Abreu" de Las Villas mediante un sistema local de indicadores de sostenibilidad ambiental [en línea]. Tesis Doctoral. Universidad Central "Marta Abreu" de Las Villas, Cuba, 2011. [Consulta 25 enero 2019]. Disponible en: http://dspace.uclv.edu.cu:8089/handle/123456789/6849 
LÓPEZ MOREDA, L. J. (2010). Tecnología para la Evaluación del Desempeño Ambiental de empresas hoteleras basada en índices de riesgo. Caso- Varadero [disco]. Tesis Doctoral. Universidad de Matanzas "Camilo Cienfuegos". Cuba.

MEDEL GONZÁLEZ, F. et al. (2015). Procedimiento para la evaluación del desempeño ambiental: aplicación em el sector energético cubano. Gestão \& Produção [en línea]. 22(3), 463-479 [Consulta 25 enero 2019]. Disponible en: http://www.scielo.br/scielo.php?pid=S0104$530 \times 2015000300463 \&$ script=sci_abstract\&tlng=es

MIRANDA CUELLAR, R. L., y FONDEN CALZADILLA, J. C. (2019). La evaluación del medio ambiente [en línea]. La Habana. Editorial Cujae. [Consulta 24 de junio 2019]. ISBN: 978959-261-593-9. Disponible en: https://www.researchgate.net/publication/335703654_La_ evaluacion_del_medio_ambiente/stats

MIRANDA CUÉLLAR, RL., PELL DEL RÍO, SILVIA MIRIAM. y FERNÁNDEZ OLIVERA, J. (2016). Proceso de evaluación del desempeño ambiental basada en indicadores sintéticos en Cuba. Revista Dilemas Contemporáneos: Educación, Política y Valores [en línea]. 4(1), 954-979. [Consulta 17 de septiembre 2018]. Disponible en: http://www. dilemascontemporaneoseducacionpoliticayvalores.com

OCHOA AVILA, M. B. (2014). Tecnología para la gestión ambiental integral en instituciones escolares. Aplicación en Holguín [disco]. Tesis Doctoral. Instituto Superior de Tecnologías y Ciencias Aplicadas. La Habana.

OFICINA NACIONAL DE NORMALIZACIÓN. ONN (2005). NC ISO 14031:2005. Gestión ambiental. Evaluación del desempeño ambiental. Directrices. La Habana. Cuba.

OFICINA NACIONAL DE NORMALIZACIÓN. ONN (2015). Sistemas de gestión de la calidad - Requisitos NC ISO 14001, La Habana, Cuba.

OLIVEIRA, J, et al. (2016). Guidelines for the integration of EMS based in ISO 14001 with Cleaner Production. Production [en línea]. 26(2), 273-284. [Consulta 17 de septiembre 2018]. Disponible en: http://dx.doi.org/10.1590/0103-6513.160214

ONU. (1992). Declaración de Rio sobre el Medio Ambiente y el Desarrollo [en línea] [Consulta 09 abril 2019]. Disponible en: http://www.unesco.org/education/pdf/RIO_S.PDF

ONU (2002). Informe de la Cumbre Mundial sobre el Desarrollo Sostenible [en línea]. Johannesburgo, Sudáfrica. [Consulta 09 abril 2019]. Disponible en: https://unctad.org/es/ Docs/aconf199d20_sp.pdf

ONU, CEPAL/OCDE. (2017). Evaluaciones del desempeño ambiental: Perú [en línea]. Santiago. Chile. [Consulta 09 abril 2019]. Disponible en: https://repositorio.cepal.org/ bitstream/handle/11362/42527/S1600240_es.pdf?sequence=1\&isAllowed=y

ONU. (2004). Programa de las Naciones Unidas para el Medio Ambiente/Banco Mundial /Universidad de Costa Rica. Iniciativa Latinoamericana y Caribeña para el Desarrollo Sostenible: Indicadores de seguimiento [en línea]. [Consulta 29 marzo 2019]. Disponible en: https://wedocs.unep.org/bitstream/handle/20.500.11822/9377/-Iniciativa_ Latinoamericana_y_Caribe\%C3\%B1a_para_el_Desarrollo_Sostenible_Indicadores_de_ segu. .pdf? sequence=2\&amp\%3BisAllowed $=$. 
ONU. (2018). La Agenda 2030 y los Objetivos de Desarrollo Sostenible: una oportunidad para América Latina y el Caribe [en línea]. Santiago. Chile. [Consulta 09 abril 2019]. Disponible en: https://www.cepal.org/es/publicaciones/40155-la-agenda-2030-objetivos-desarrollosostenible-oportunidad-america-latina-caribe

ONU. Asamblea General (2012). El futuro que queremos [en línea]. Nueva York. [Consulta 09 abril 2019]. Disponible en: https://www.un.org/en/ga/search/view_doc.asp?symbol=A/ RES/66/288\&Lang=S

ONU. Asamblea General (2015). Transformar nuestro mundo: La Agenda 2030 para el Desarrollo Sostenible [en línea]. Nueva York. [Consulta 09 abril 2019]. Disponible en: https:// unctad.org/meetings/es/SessionalDocuments/ares70d1_es.pdf

ONU. PNUMA (2012). GEO 5. Perspectivas del Medio Ambiente Mundial. Resumen para responsables de políticas [en línea] [Consulta 09 abril 2019]. Disponible en: https://wedocs. unep.org/bitstream/handle/20.500.11822/8057/-GEO-5\%20summary\%20for\%20policy\%20 makers-2012GEO5_SPM_Spanish.pdf?sequence=11\&isAllowed=y

Oregi, A. \& Galera, J. (2013). Índice de Rendimiento Ambiental (EPI) 2013. Euskadi [en línea]. [Consulta 29 marzo 2019]. Disponible en: www.ihobe.net

PARTIDO COMUNISTA DE CUBA. Comité Central. (2017) Documentos del 7mo. Congreso del Partido aprobados por el III Pleno del Comité Central del PCC el 18 de mayo de 2017 y respaldados por la Asamblea Nacional del Poder Popular el 1 de junio de 2017 [disco]. Septiembre 2017, La Habana: UEB Gráfica. Empresa de Periódicos.

PEREVOCHTCHIKOVA, M. (2013). La evaluación del impacto ambiental y la importancia de los indicadores ambientales. Gestión y política pública [en línea]. 22(2), 283-312. [Consulta 09 abril 2019]. Disponible en: http://www.scielo.org.mx/pdf/gpp/v22n2/v22n2a1.pdf

PÉREZ GARCÍA, W. (2013). Modelo de gestión integrada de la calidad y del medio ambiente en los órganos cubanos de gobierno local [disco]. Tesis Doctoral. Instituto Superior de Tecnologías y Ciencias Aplicadas. La Habana. Cuba.

RODRÍGUEZ GUERRA, L.C. y RÍOS-OSORIO, L.A. (2016). Evaluación de sostenibilidad con metodología GRI [en línea]. Dimensión Empresarial 14(2), 73-89. [Consulta 25 de marzo 2019]. Disponible en: http://dx.doi.org/10.15665/rde.v14i2.659.

SIEMENS GAMESA. (2018). Informe de Sostenibilidad 2018 [en línea]. [Consulta 29 de marzo 2019]. Disponible en: https://www.siemensgamesa.com/es-es/-/media/siemensgamesa/ downloads/es/sustainability/siemens-gamesa-sustainability-report-2018-es.pdf

STANTEC. (2017) SUSTAINABILITY REPORT [en línea]. [Consulta 29 de marzo 2019]. Disponible en: https://www.stantec.com/content/dam/stantec/files/PDFAssets/2018/stn-2017sustainability-report.pdf

TOYOTA MATERIAL HANDLINGEUROPE. (2018). Informe de Sostenibilidad 2018 [en línea]. [Consulta 29 marzo 2019]. Disponible en: https://toyota-forklifts.es/globalassets/2018_tmheinforme-sostenibilidad-v2.pdf

ULLOA ENRÍQUEZ, M. A. (2012). Procedimiento para la integración de los riesgos 
ambientales y de seguridad y salud ocupacional al sistema de gestión de calidad. Caso de estudio Empresa de elaborados Cárnicos S.A de Latacunga, Ecuador [disco]. Tesis Doctoral. Universidad de Holguín. Cuba.

WONG, C. W., LAI, K. H., SHANG, K. C., LU, C. S. y LEUNG, T. K. P. (2012). Green operations and the moderating role of environmental management capability of suppliers on manufacturing firm performance. International Journal of Production Economics [en línea]. 140(1), 283-294. [Consulta 25 de enero 2019]. Disponible en: https://doi.org/10.1016/j.ijpe.2011.08.031

ZÚÑIGA IGARZA, L. M. (2011). Metodología: Gestión ambiental urbana de recursos construidos de valor patrimonial. Aplicación en Gibara, Holguín [disco]. Tesis Doctoral. Instituto Superior de Tecnologías y Ciencias Aplicadas Holguín. Cuba. 